\title{
Oxidative stress and antioxidant mechanisms in human body
}

\begin{abstract}
The present review aims to high light on the oxidative stress, and prevention by internal antioxidants and external antioxidants by some natural products possessing antioxidant properties. Oxidative stress occurs when the balance between reactive oxygen species (ROS) formation and detoxification favors an increase in ROS levels, leading to disturbed cellular function. ROS causes damage to cellular macromolecules causing lipid peroxidation, nucleic acid, and protein alterations. Their formation is considered as a pathobiochemical mechanism involved in the initiation or progression phase of various diseases such as atherosclerosis, ischemic heart diseases, diabetes, and initiation of carcinogenesis or liver diseases. In order to maintain proper cell signaling, it is likely that a number of radical scavenging enzymes maintain a threshold level of ROS inside the cell. However, when the level of ROS exceeds this threshold, an increase in ROS production may lead to excessive signals to the cell, in addition to direct damage to key components in signaling pathways. ROS can also irreversibly damage essential macromolecules. Protein-bound thiol and non-proteinthiol are the major cytosolic low molecular weight sulfhydryl compound that acts as a cellular reducing and a protective reagent against numerous toxic substances including most inorganic pollutants, through the $-\mathrm{SH}$ group. Hence, thiol is often the first line of defense against oxidative stress. Flavonoids have been found to play important roles in the non-enzymatic protection against oxidative stress, especially in the case of cancer. Flavonoids have occurred widely in tea, fruit, red wine, vegetables, and cocoas. Flavonoids, including flavones, flavanone, flavonols, and isoflavones, are polyphenolic compounds which are widespread in foods and beverages, and possess a wide range of biological activities, of which anti-oxidation has been extensively explored. It can be concluded that oxidative stress causes irreversible damage in cellular macromolecules that leads to initiation of various diseases such as atherosclerosis, ischemic heart diseases, liver diseases, diabetes, and initiation of carcinogenesis. Antioxidants inhibit reactive oxygen species production and scavenging of free radicals. Therefore, the review recommends that high consumption of natural foods that are rich in antioxidants will provide more protection against toxic agents and related diseases.
\end{abstract}

Volume 6 Issue I - 2019

\author{
Almokhtar A Adwas,' Ata Sedik Ibrahim \\ Elsayed, ${ }^{2}$ Azab Elsayed Azab, ${ }^{3}$ Fawzia \\ Amhimmid Quwaydir ${ }^{4}$ \\ 'Department of Pharmacology, Faculty of Medicine, Sabratha \\ University, Libya \\ 2Department of Basic Medical Sciences, Inaya Medical College, \\ Saudi Arabia \\ ${ }^{3}$ Department of Physiology, Faculty of Medicine, Sabratha \\ University, Libya \\ ${ }^{4}$ Department of Zoology, Faculty of Science, Sabratha University, \\ Libya
}

Correspondence: Azab Elsayed Azab, Head of Physiology Department, Faculty of Medicine, Sabratha University, Sabratha, Libya,Email azabelsaied@yahoo.com

Received: November 27, 2018 | Published: February 21, 2019

Keywords: antioxidants, cancer, flavonoids, oxidative stress

\section{Introduction}

Today, oxidative stress has attracted the attention of researchers. An imbalance between free radicals and antioxidants leads to oxidative damage of proteins, fat, nucleic acids, and carbohydrates. ${ }^{1,2}$ Antioxidants have protected the body from the harmful effect of the free radicals.,3 Endogenous antioxidants defense against the reactive oxygen species are strengthened by natural antioxidants that strengthen them and restore the optimal balance by neutralizing the ROS. ${ }^{2,4-7}$ Traditional medicine has been used a number of natural and herbal products to treat a variety of diseases including malignant tumors. ${ }^{7,8}$ Many herbal plants have proven their ability to resist cancerous activities. Some previous studies have shown that herbal drug might have antitumor effect by promoting the immune system, including cell differentiation, inducing apoptosis of cancer cells and inhibiting telomerase activities. ${ }^{7,9}$

\section{Objectives}

The present review aims to high light on the oxidative stress, and its prevention by internal antioxidants and external antioxidants by some natural products possessing antioxidant properties.

\section{Free radicals and mechanism of their destructive effects}

Free radical are molecules which contain unpaired electron in the outer orbitals, and is highly reactive in the body by oxidizing (removing an electron from) other atoms, or sometimes reducing (donating their electron to) other atoms. The major source of reactive oxygen species are mitochondria, produced by electron transport chain in aerobic respiration as byproducts. Although most electrons reach the third pump of the electron transport system, about $1 \%$ to $3 \%$ reacts with oxygen prematurely to form the superoxide radical. ${ }^{10}$ The production of free radicals makes important physiological functions in the body, one of these functions is the production of superoxide and nitric oxide by neutrophils and macrophages which aids in phagocytosis process and help these cells in destroying bacteria. The superoxide radicals in phagocytic cells can be thought of as nonselective antibiotics, killing any infecting bacteria (as well as the neutrophils) and perhaps also injuring surrounding tissue cells, as these radicals contribute to the inflammation reaction, these free radicals also promote cellular proliferation (mitotic division) of fibroblasts, so that scar tissue can form and stimulate proliferation of lymphocytes in the process of 
clone production. No has also effects on promoting relaxation of vascular smooth muscle which causes vasodilation and an increase in the blood can flow to the site of the inflammation. ${ }^{10}$

The generation of ROS and RNS by endogenous and exogenous sources. The endogenous generation of these species by inflammation mechanisms and activation of immune cells, sever exercise, ischemia, mental activity stress, cancerous and infectious diseases, and aging. Exogenous sources of ROS result from the pollution of water and air and water, alcohol drinking, smoking, some drugs, heavy metals, certain drugs (tacrolimus and cyclosporine), radiations, cooking and some solvents as benzene. These compounds are decomposed into ROS after they penetrate the body, ${ }^{11}$ The damaging effects of ROS on cellular macromolecules such as proteins, lipids, and nucleic acid are causing alterations in proteins, and nucleic acid. The formation of these free radicals leads to the initiation and progression of many diseases such as diabetes, heart diseases, atherosclerosis, liver diseases and cancers. ${ }^{12}$

Oxidative stress is linked to altered redox regulation of cellular signaling pathways and the formation of many types of cancer cells and oncogenic stimulation. ${ }^{13}$ It appears that the DNA damage and activation of ROS to AP-1 and NF-kB pathways of signal transduction leads to the transcription of genes involved in cell growth regulation and initiation of cancerous conditions. Lipid peroxidation products are formed with the abstraction of a hydrogen atom from an unsaturated fatty acid. ${ }^{12}$ The lipid peroxidation process influences membrane fluidity as well as the integrity of biomolecules associated with the membrane (membrane bound proteins or cholesterol). These highly oxidizable lipids may then, in turn, attack nearby proteins causing the formation of an excess of protein carbonyls. ${ }^{14}$ A major development over the past two decades has been the realization that free radical mediated peroxidation of membrane lipids and oxidative damage of DNA are associated with a variety of chronic health problems, ${ }^{15}$ such as cancer,atherosclerosis, ${ }^{16}$ neurodegenerative diseases, ${ }^{17}$ and aging. ${ }^{18}$ Therefore, inhibition of oxidative damage by supplementation of antioxidants becomes an attractive therapeutic strategy to reduce the risk of these diseases. ${ }^{19}$

\section{Antioxidant enzymes and their action mechanisms}

In order to maintain proper cell signaling, it is likely that a number of radical scavenging enzymes maintain a threshold level of ROS inside the cell. However, when the level of ROS exceeds this threshold, an increase in ROS production may lead to excessive signals to the cell, in addition to direct damage to key components in signaling pathways. Oxidative stress occurs when the balance between ROS formation and detoxification favors an increase in ROS levels, leading to disturbed cellular function. ROS can also irreversibly damage essential macromolecular targets such as DNA, protein and lipids, which may initiate carcinogenesis. ${ }^{20}$ Therefore, the concentrations of ROS have to be controlled by several defense mechanisms, which involve also a number of antioxidant and detoxifying enzymes.

An antioxidant is a molecule which has the ability to prevent or slow the oxidation of macromolecules. The role of antioxidants is to lower or terminate these chain reactions by removing free radicals or inhibiting other oxidation reactions by being oxidized themselves. So, antioxidants are often reducing agents such as polyphenols or thiols. ${ }^{21}$

Although oxidation reactions are vital for cells, they have damaging effects; hence, plants and animals contain various antioxidants, such as vitamins $\mathrm{C}$ and $\mathrm{E}$ and glutathione, as well as different enzymatic systems which catalyze the antioxidants reactions as catalase, superoxide dismutase (SOD) and peroxidases. The defects in or inhibition of these antioxidant enzymes will lead to oxidative stress and may damage and lyse the cells. ${ }^{11}$ The mechanisms which followed by antioxidant defense are: 1) Blocking of free radicals production of 2) oxidants Scavenging 3) The converting toxic free radicals into less toxic substances 4) Blocking the production of secondary toxic metabolites and mediators of inflammation 5) Blocking of the chain propagation of the secondary oxidants 6) Repairing the injured molecules 7) Initiation and enhancing the endogenous antioxidant defense system. All of these defense mechanisms act hand by hand for protection of the body from oxidative stress. The antioxidant systems in the human body consist of powerful non-enzymatic and enzymatic antioxidants. $^{12}$

The antioxidant enzymes in all body cells consist of three major classes of antioxidant enzymes which are the catalases, superoxide dismutases (SOD), and glutathione peroxidases (GPX), all of these, play crucial roles in maintaining homeostasis into cells. The induction of these enzymes reflects a specific response to pollutant oxidative stress. ${ }^{22}$ The role of SOD is to scavenge superoxide radicals and converting them to $\mathrm{H}_{2} \mathrm{O}_{2}{ }^{23}$ The role of GPx is achieved by the reduction of hydrogen peroxide, lipid hydroperoxides and other organic hydroperoxides. ${ }^{24}$ Glutathione-S-transferases (GST) represent a major group of detoxifying enzymes, ${ }^{25}$ which form a family of multifunctional proteins involved in the cellular detoxification of cytotoxic and genotoxic compounds and in the protection of tissues against oxidative damage. ${ }^{26,27}$ Besides certain roles in the endogenous metabolism, these enzymes are associated with the detoxification of xenobiotics such as drugs, carcinogens, and environmental pollutants in man and animals, and with pesticide and herbicide resistance in insects and plants. ${ }^{28}$ Some studies have shown, that GPx, ${ }^{29}$ and GST are inactivated by hydroperoxides, which exert their toxicity either directly by oxidation of SH groups of proteins or indirectly by hydroxyl radicals formation. It is known from the literature that a significant number of the GST isoenzymes also exhibit GPx activity and catalyze the reduction of organic hydroperoxides to their corresponding alcohols. ${ }^{31,32}$ Superoxide dismutase catalyzes the breakdown of the superoxide anion into oxygen and hydrogen peroxide. ${ }^{33}$ It removes $\mathrm{O}_{2}$ by catalyzing a dismutation reaction. In the absence of this enzyme, this reaction becomes very slow. ${ }^{34}$

Catalase $\left(\mathrm{H}_{2} \mathrm{O}_{2}\right.$ oxidoreductase $)$ is composed of four polypeptide chains, each chain is over 500 amino acids long, and contains four porphyrin heme (iron) groups allowing the enzyme to react with the $\mathrm{H}_{2} \mathrm{O}_{2}$. The enzymatic activity modes of catalase in decomposing $\mathrm{H}_{2} \mathrm{O}_{2}$ are the catalytic mode of activity $\left(2 \mathrm{H}_{2} \mathrm{O}_{2}+\mathrm{O}_{2}+2 \mathrm{H}_{2} \mathrm{O}\right)$ and the peroxidatic mode of activity $\left(\mathrm{H}_{2} \mathrm{O}_{2}+\mathrm{AH}_{2} \mathrm{~A}+2 \mathrm{H}_{2} \mathrm{O}\right)$. The turnover rate of catalase is the highest among all of the other antioxidant enzymes. Decomposition of $\mathrm{H}_{2} \mathrm{O}_{2}$ by the catalytic activity of catalase follows the fashion of a first-order reaction and its rate is dependent on the concentration of $\mathrm{H}_{2} \mathrm{O}_{2}{ }^{35}$ Catalase is cofactor oxidized by one molecule of hydrogen peroxide and transfer the bound oxygen to another molecule of the substrate. ${ }^{36}$

Catalase exists in both eukaryotic and bacterial cells. Most of them are located in an oxidative particle of all types of mammalian cells except red blood cells where various $\mathrm{H}_{2} \mathrm{O}_{2}$ oxidases were created. Since $\mathrm{H}_{2} \mathrm{O}_{2}$ acts as a substrate for a specific reaction that generates highly hydroxyl radical, it is believed that the primary role of catalase in cellular antioxidant defense mechanisms is to reduce the accumulation of $\mathrm{H}_{2} \mathrm{O}_{2} \cdot{ }^{37}$ The role of catalase in protecting cells and tissues from 
oxidation has been studied extensively. The overexpression of catalase makes cells more resistant to $\mathrm{H}_{2} \mathrm{O}_{2}$ toxicity and oxidative-mediated damage. In genetically modified mice, overexpressing catalase is protected against myocardial infarction after giving rats adriamycin and developing high blood pressure after treatment with paraffin or angiotensin. Patients with naturally occurring catalase deficiency, except for an increased tendency to progressive oral gangrene development due to tissue damage from $\mathrm{H} 2 \mathrm{O} 2$ resulting from bacteria producing peroxides such as streptococcus and pneumococcus, as well as phagocytic cells in bacterial sites. ${ }^{38}$ Thioredoxin system includes thioredoxin protein and thioredoxin reductase, ${ }^{39}$ Thioredoxin-related proteins are present in all living organisms. It scavenges the ROS. ${ }^{40}$

Glutathione system includes glutathione $\mathrm{S}$-transferases, glutathione peroxidases, and glutathione reductase. Glutathione S-transferases are another class of enzymatic antioxidants that catalyze the breakdown of lipid peroxides. Glutathione peroxidase shows a high activity with hydrogen peroxide and organic hydroperoxides. ${ }^{41}$ These enzymes help in detoxification mechanism..$^{42}$

Glutathione reductase (GR) catalyzes the reduction of oxidized glutathione (GSSG) to reduced glutathione (GSH). This enzyme enables the cell to sustain adequate levels of cellular GSH. Due to its importance, the enzyme was purified from a number of plants, animals and microorganism sources and studied in an attempt to identify and clarify its structure, molecular properties and kinetic mechanism. ${ }^{43}$ Its kinetic mechanism is known to be a ping-pong/sequentially ordered model. GR is a flavoprotein that contains two FAD molecules as a prosthetic group, which is reducible by NADPH. GR is one of the thermostable enzymes. GR belongs to the defense system protecting the organism against chemical and oxidative stress. Deficiency of GR is characterized by hemolysis due to increased sensitivity of erythrocyte membranes to $\mathrm{H}_{2} \mathrm{O}_{2}$ and contributes to oxidative stress which plays a key role in the pathogenesis of many diseases. ${ }^{44}$

\section{Non-enzymatic antioxidants and their action mechanisms}

Protein-bound thiol and nonprotein-thiol are acting as a cellular reducing and a protective agent against most inorganic pollutants, through the $-\mathrm{SH}$ group. ${ }^{32}$ Hence, the thiol is often the first line of defense against oxidative stress. The thiol levels can be increased due to an adaptive mechanism to slight oxidative stress through an increase in its synthesis; however, a severe oxidative stress may decrease thiol levels due to loss of adaptive mechanisms.

Glutathione is a cellular antioxidant which plays a central role in maintaining the cells redox state. ${ }^{44}$

Ascorbic acid is an antioxidant found in both plants and animals but it must be obtained from the diet in humans because it cannot be synthesized. It can reduce and neutralize reactive oxygen species. ${ }^{43}$ Vitamin $\mathrm{E}$ has been found to protect the cell membranes from oxidation by removing the free radical intermediates and reacting with the lipid radicals. ${ }^{45}$ Beta-carotene has potent the antioxidant properties by removing a singlet oxygen thus protects against a free radical attack. They are present in liver, egg yolk, milk, butter, spinach, carrots, tomato and grains. ${ }^{43}$

\section{Natural antioxidants}

The protective effects of natural antioxidants has received more attention against free radical induced toxicities ${ }^{46}$ Flavonoids play an important role in the protection against oxidative stress, ${ }^{47,48}$ especially in the case of cancer. Flavonoids are found widely in vegetables, red wine, fruit, cocoas, and tea.9-51 Flavonoids are widespread in beverages and foods which possess a wide range of biological activities $^{52}$ of which antioxidation has been extensively explored. ${ }^{53-60}$ Natural antioxidants strengthen the endogenous antioxidants defenses from ROS and restore the optimal balance by neutralizing reactive species. ${ }^{4,6}$ The antioxidant activities of phenolics are related to a number of different mechanisms, such as free radical-scavenging, hydrogendonation, singlet oxygen quenching, metal ion chelating, and acting as a substrate for radicals such as superoxide and hydroxyl. ${ }^{7,32}$ Azab \& Albash $^{7}$ concluded that administration of Curcuma longa, Trigonella foenumgraecum, Allium sativum, Coffea arabica, Petroselinum crispum, Olea europaea leaves, and Mentha piperita were showed a remarkable hepatic protection against hepatotoxic agents, which may be due to its antioxidant properties of these medicinal plants and herbs. Therefore, Human expose to hepatotoxic agents and patients with hepatic disorders should be advised to take these medicinal plants and herbs.

Natural antioxidants have a variety of biochemical actions such as inhibition of the production of ROS and scavenging of free radicals. 2,61,62 Azab et al., ${ }^{2}$ reported that consumption of fenugreek, curcumin, peppermint, parsley, rosemary, garlic, pomegranate, sesame, and propolis were shown protective effects against renal diseases and nephrotoxic agents induced renal dysfunctions in experimental animals and human. The nephroprotective effect may be due to the inhibition of tissue lipid peroxidation and enhancement of antioxidant activity. Therefore, the study suggested that these antioxidants may be useful for the persons who expose to nephrotoxic agents and patients suffer from renal diseases. The protective effects may be due to the presence of benzoquinones, flavonoids, flavonol glycosides, alkaloids, carotenoids, catechols, glycosides, steroid glycosides, terpenoids, glycoalkaloids, mono, di, and triterpenes, saponins, polyphenols, and sterols in these medicinal plants and herbs. ${ }^{2,61,63}$

\section{Conclusion}

It can be concluded that oxidative stress causes irreversible damage in cellular macromolecules that leads to initiation of various diseases such as atherosclerosis, ischemic heart diseases, liver diseases, diabetes, and initiation of carcinogenesis. Antioxidants inhibit of reactive oxygen species production and scavenging of free radicals. Therefore, the review recommends that high consumption of natural foods that are rich in antioxidants will provide more protection against toxic agents and related diseases.

\section{Acknowledgments}

None.

\section{Conflicts of interest}

The author declares there are no conflicts of interest.

\section{References}

1. Prior R,Cao G. Antioxidant Capacity and Polyphone Compounds of Teas. PSEBM. 1999;220:255-261

2. Azab AE, Albasha MO, Elsayed ASI. Prevention of nephropathy by some natural sources of antioxidants. Yangtze Medicine. 2017;1:235266. 
3. Robinson EE, Maxwell SR, Thorpe GH. An Investigation of Antioxidan Activity of Black Tea, using Enhanced Chemiluminescence. Free Radic Res. 1997;26:291-300.

4. Al-Mamary M, Al-Meeri A, Al-Habori M. Antioxidant activities and total phenolics of different types of honey. Nutr Res. 2002;22:10411047.

5. Albasha MO, Azab AE. Effect of cadmium on the liver and amelioration by aqueous extracts of fenugreek seeds, rosemary, and cinnamon in Guinea pigs: Histological and biochemical study. $J$ Cell Biol. 2014;2:34-44.

6. Fetouh FA, Azab AE. Ameliorating effects of curcumin and propolis against the reproductive toxicity of gentamicin in adult male Guinea pigs: Quantitative analysis and morphological study. Amer J Life Sci. 2014;2(3):138-149.

7. Azab AE, Albasha MO. Hepatoprotective effect of some medicina plants and herbs against hepatic disorders induced by hepatotoxic agents. J Biotechnol Bioeng. 2018;2(1):8-23.

8. Yin $\mathrm{X}$, Zhou J, Jie C, et al. Anticancer activity and mechanism of Scutellaria barbata extract on human lung cancer cell line. Life Sci. 2004;75:2233-2244

9. Gill CL, Boyed A, McDermott E, et al. Potential anticancer effects of virgin olive oil phenols on colorectal carcinogenesis models in vitro. Int J Cancer. 2005;117(1): 1-7.

10. Fox SI. Human Physiology. 16th Ed. McGraw-Hill Education. 2017. p. 666-670.

11. Valko M, Leibfritz D, Moncol J, et al. Free radicals and antioxidants in normal physiological functions and human disease. Int J Biochem Cell Biol. 2007;39(1):44-84.

12. Halliwell B. Biochemistry of oxidative stress. Biochem Soc Trans 2007;35:1147-1150.

13. Miranda-Vilelaa AL, Portilhoa FA, de Araujoa V, et al. The protective effects of nutritional antioxidant therapy on Ehrlich solid tumorbearing mice depend on the type of antioxidant therapy chosen: histology, genotoxicity and hematology evaluations. J Nutr Biochem. 2011;22(11):1091-1098.

14. Almroth BC, Sturve J, Berglund A, et al. Oxidative damage in eelpout (Zoarces viviparus), measured as protein carbonyls and TBARS, as biomarkers. Aquatic Toxicol. 2005;73:171-180.

15. Mukhtar H, Ahmad N. Tea polyphenols: prevention of cancer and optimizing health. Am J Clin Nutr. 2000;71(1):1698s-1702s.

16. Miura Y, Chiba T, Tomita I, et al. Tea catechins prevent the development of atherosclerosis in apoprotein E-deficient mice. J Nutr. 2001;131(1):27-32.

17. Rafalowska U, Liu GJ, Floyd RA. Peroxidation induced changes in synaptosomal transport of dopamine and gamma- minobutyric acid. Free Radic Biol Med.1989;6:485-492.

18. Finkel T, Holbrook NJ. Oxidants, oxidative stress and the biology of ageing. Nature. 2000;408:239-247.

19. Dai F, Miao Q, Zhou B, et al. Protective effect of flavonols and their glycosides against free radical-induced oxidative hemolysis of red blood cells. Life Sci. 2006;78:2488-2493.

20. Imlay JA. Pathways of oxidative damage. Annu Rev Microbiol. 2003;57:395-418.

21. Duarte TL, Lunec J. When is an antioxidant not an antioxidant? A review of novel actions and reactions of vitamin C. Free Radic Res. 2005;39(7):671-686
22. Cheung CC, Zheng GJ, Li AM, et al. Relationship between tissue concentrations of polycyclic aromatic hydrocarbons and antioxidative responses of marine mussels, Perna viridis Aquat Toxicol. 2001;52:189_ 203.

23. McCord JM, Fridovich I. Superoxide dismutase. An enzymic function for erythrocuprein (hemocuprein). J Biol Chem. 1969;244:6049-6055.

24. Tappel ME, Chaudiere J, Tappel AL. Glutathione peroxidase activities of animal tissues. Comp Biochem Physiol.1982;73B:945-949.

25. Hayes JD, Pulford DJ. The glutathione-S-transferase supergene family: regulation of resistance. Crit Rev Biochem Mol Biol. 1995;30:445-600.

26. Mannervik B, Danielson UH. Glutathione transferases-structure and catalytic activity. CRC Crit Rev Biochem.1988;23:283-337.

27. Pickett CB, Lu AY. Glutathione-S-transferases: gene structure, regulation, and biological function. Annu Rev Biochem. 1989;58:743764.

28. Hayes JD, Pickett CB, Mantle et al. Glutathione-S-transferases and drug resistance. London: Taylor \& Francis. 1990. p. 3-16.

29. Pigeolet E, Corbisier P, Houbion A, et al. Glutathione peroxidase, superoxide dismutase and catalase inactivation by peroxides and oxygen derived free radicals. Mech Ageing Dev. 1990;51:283-297.

30. Shen H, Tamai K, Satoh K, et al. Modulation of class Pi glutathione transferase activity by sulfhydryl group modification. Arch Biochem Biophys. 1991;286:178-182.

31. Prohaska JR. Changes in $\mathrm{Cu}, \mathrm{Zn}$-superoxide dismutase, cytochrome $\mathrm{C}$ oxidase, glutathiohne peroxidase and glutathione transferase activities in copper-deficient mice and rats. $J$ Nutr.1991;121:355-363.

32. Mosialou E, Ekström G, Adang AE, et al. Evidence that rat liver microsomal glutathione transferase is responsible for glutathionedependent protection against lipid peroxidation. Biochem Pharmacol. 1993;45:1645-1651.

33. Zelko IN, Mariani TJ, Folz RJ. Superoxide dismutase multigene family: a comparison of the CuZn-SOD (SOD1), Mn-SOD (SOD2), and ECSOD (SOD3) gene structures, evolution, and expression. Free Radic Biol Med. 2002;33(3):337-349.

34. Nozik-Grayck E, Suliman H, Piantadosi C. Extracellular superoxide dismutase. Int J Biochem Cell Biol. 2005;37(12):2466-2471.

35. Berg JM, Tymoczko JL, Stryer L. Biochemistry. 5th ed. Freeman WH and Co, New York. 2002. P. 205-206.

36. Hiner AN, Raven EL, Thorneley RN, et al. Mechanisms of compound I formation in heme peroxidases. J Inorg Biochem. 2002;91(1):27-34.

37. Ho YS, Xiong Y, Ma W, et al. Mice lacking catalase develop normally but show differential sensitivity to oxidant tissue injury. $J$ Biol Chem. 2004;279:32804-32812.

38. Yang H, Shi MJ, Van Remmen H, et al. Reduction of pressor response to vasoconstrictor agents by overexpression of catalase in mice. $\mathrm{Am} \mathrm{J}$ Hypertens. 2003;16(1):1-5.

39. Nordberg J, Arner ES. Reactive oxygen species, antioxidants, and the mammalian thioredoxin system. Free Radic Biol Med. 2001;31(11):1287-1312.

40. Mustacich D, Powis G. Thioredoxin reductase. Biochem J. 2000;346(1):1-8.

41. Sharma R, Yang Y, Sharma A, et al. Antioxidant role of glutathione S-transferases: protection against oxidant toxicity and regulation of stress-mediated apoptosis. Antioxid Redox Signal. 2004;6(2):289-300.

42. Hayes J, Flanagan J, Jowsey I. Glutathione transferases. Annu Rev Pharmacol Toxicol. 2005;45:51-88. 
43. Linster CL, Van Schaftingen E. Vitamin C: Biosynthesis, recycling and degradation in mammals. FEBS J. 2007;274(1):1-22.

44. Ulusu NN, Tandoğan B. Purification and kinetic properties of glutathione reductase from bovine liver. Mol Cell Biochem. 2007;303(1-2):45-51.

45. Sen C, Khanna S. Tocotrienols RS. Vitamin E beyond tocopherols. Life Sci. 2006;78(18):2088-2098.

46. Frei B, Higdon J. Antioxidant activity of tea polyphenols in vivo: evidence from animal studies. J Nutr. 2003;133:3275-3284.

47. Okada K, Wangpoengtrakul C, Tanaka T, et al. Curcumin and especially tetrahydrocurcumin ameliorate stress-induced renal injury in mice. $J$ Nutr. 2001;131:2090-2095

48. Babich H, Gold T, Gold R. Mediation of the in vitro cytotoxicity of green tea and black tea polyphenols by cobalt chloride. Toxicol Lett. 2005; 155:195-205.

49. Arts IC, Hollman PC, Kromhout D. Chocolate as a source of tea flavonoids. Lancet. 1999;61:354-488.

50. Bearden ZM, Pearson D, Rein D. Potential cardiovascular health benefits of procyanidins present in chocolate and cocoa; in Caffeinated Beverages: Health Benefits. In: Parliament TH, editor. Oxford University Press, Washington DC, USA. 2000. p. 177-186.

51. Matito C, Mastoraku F, Centelles ZJ, et al. Antiproliferative effect of antioxidant polyphenols from grape in murine Hep1c1c7. Eur J Nutr. 2003;42:43-49.

52. Harborne JB, Williams CA. Advances in flavonoid research since 1992. Phytochemistry. 2000;55:481-504.

53. Bors W, Michel C, Saran M. Flavonoid antioxidant-rate constants for reactions with oxygen radicals. Meth Enzymol. 1994;234:420-429.

54. Terao J, Piskula M, Yao Q. Protective effect of epicatechin, epicatechin gallate and quercetin on lipid peroxidation in phospholipids bilayers. Archives of Biochemistry and Biophysics. 1994;308:278-284.
55. Ioku K, Tsushida T, Takei $\mathrm{T}$, et al. Antioxidative activity of quercetin and quercetin monoglucosides in solution and phospholipid bilayers. Biochimica et Biophysica Acta.1995;1234:99-104.

56. CroftKD. The chemistry and biological effects of flavonoids and phenolic acids. Annals of the New York Academy of Sciences.1998;854:435442R.

57. Pietta PG. Flavonoids as antioxidants. $J$ Natural Products. 2000;63:1035-10422.

58. McPhail DB, Hartley RC, Gardner PT, et al. Kinetic and stoichiometric assessment of antioxidant of flavonoids by ESR spectroscopy. $J$ Agricul Food Chem . 2003;51:1684-1690.

59. Goupy P, Dufour C, Loonis M, et al Quantitative kinetic analysis of hydrogen transfer reactions from dietary polyphenols to the DPPH radical. J Agricult Food Chem. 2003;51:615-622.

60. Vaya J, Mahmood S, Goldblum A, et al. Inhibition of LDL oxidation by flavonoids in relation to their structure and calculated enthalpy. Phytochemistry. 2003;62:89-99.

61. Lakshmi MS, Reddy UK, Rani SRKS. A Review on Medicinal Plants for Nephroprotective Activity. Asian Journal of Pharmaceutical and Clinical Research. 2012;5:8-14.

62. Cyril DG, Landry KS, Francois KYK, et al. Evaluation of Nephroprotective Activity of Aqueous and Hydroethanolic Extracts of Trema guineensis Leaves (Ulmaceae) against Gentamicin-Induced Nephrotoxicity in Rats. International Journal of Biochemistry Research \& Review. 2016;15:1-10.

63. Sundararajan R, Bharampuram A, Koduru RA. Review on PhytoConstituents for Nephroprotective Activity. Pharm. 2014;5:160-182. 